\title{
Correction to: Elevated expression of CST1 promotes breast cancer progression and predicts a poor prognosis
}

\author{
Da-nian Dai ${ }^{1,2} \cdot$ Yan $\mathrm{Li}^{1} \cdot$ Bo Chen ${ }^{1,2} \cdot$ Yong $\mathrm{Du}^{1} \cdot$ Shi-bing $\mathrm{Li}^{1} \cdot$ Shi-xun $\mathrm{Lu}^{3} \cdot$ Zhi-ping Zhao $^{4} \cdot$ Ai-jun Zhou ${ }^{1}$. \\ Ning Xue ${ }^{1} \cdot$ Tian-liang Xia ${ }^{1} \cdot$ Mu-sheng Zeng ${ }^{1} \cdot$ Qian Zhong ${ }^{1,2} \cdot$ Wei-dong Wei ${ }^{1,2}$
}

Published online: 15 June 2019

(C) Springer-Verlag GmbH Germany, part of Springer Nature 2019

\section{Correction to: J Mol Med (2017) 95:873-886}

https://doi.org/10.1007/s00109-017-1537-1

In Fig. $7 \mathrm{f}$ the panel for c-myc of MDA-MB-468 was erroneously duplicated.

The corrected version of the figure is shown in this paper. This correction does not influence the conclusion of the study and we sincerely apologize for this oversight.

The online version of the original article can be found at https://doi.org/ 10.1007/s00109-017-1537-1

Qian Zhong

zhongqian@sysucc.org.cn

$\triangle$ Wei-dong Wei

weiwd@sysucc.org.cn

1 State Key Laboratory of Oncology in South China, Collaborative Innovation Center for Cancer Medicine, Sun Yat-Sen University Cancer Center, Guangzhou, Guangdong, China

2 Department of Breast Oncology, Sun Yat-Sen University Cancer Center, 651 East Dongfeng Road, Guangzhou 510060, China

3 Department of Pathology, Sun Yat-Sen University Cancer Center, Guangzhou, China

4 Institute of Hepatopancreatobiliary Surgery, Southwest Hospital, Third Military Medical University, Chongqing, China 
Fig. 7 Knockdown of CST1 suppresses proliferation in breast cancer cells. a Knockdown of CST1 resulted in reduced CST1 protein expression in BT-474 and MDA-MB-468 cell lines. b Cell proliferation after CST1 knockdown in BT-474 cells was measured by MTT assay. c Cell proliferation after CST1 knockdown in MDA-MB-468 cells was measured by MTT assay. d Cell colony formation was determined in BT-474 and MDA-MB-468 cells transfected with NC- or CST1targeting siRNAs. e The number of colonies after knockdown of CST1 in BT-474 and MDA-MB468 cells. f The representative pictures of proliferation-related proteins after knockdown of CST1, as determined by western blotting. Results are expressed as means $\pm \mathrm{SD}$ (error bars). * $p<$ $0.05, * * p<0.01$, and $* * * p<$ 0.001 compared to siNC a

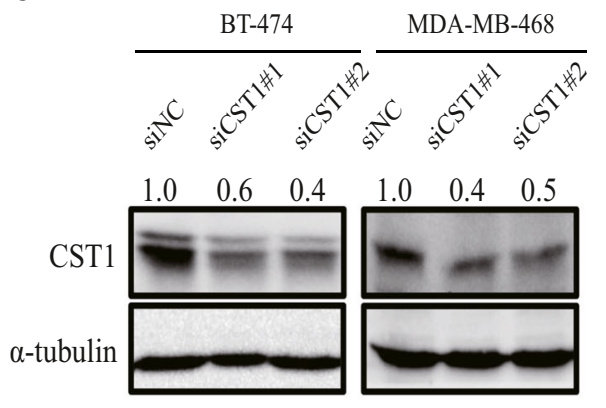

C

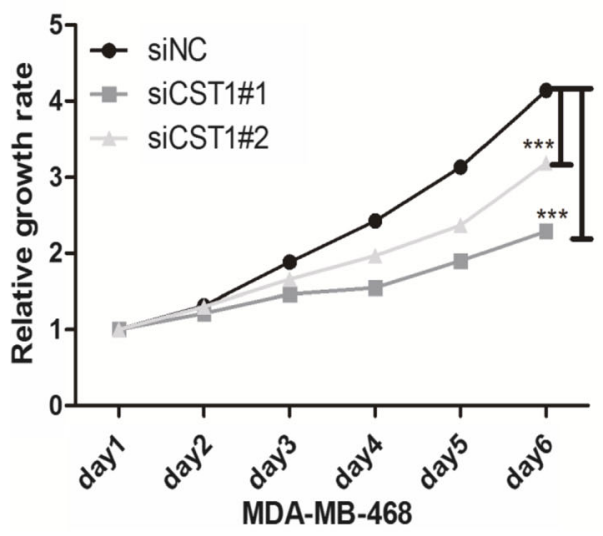

e

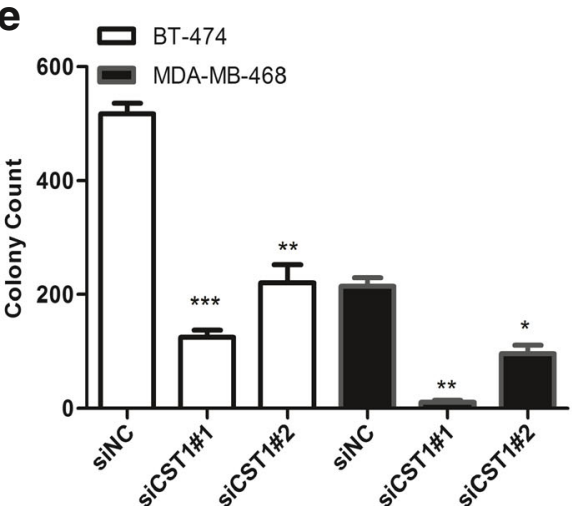

b

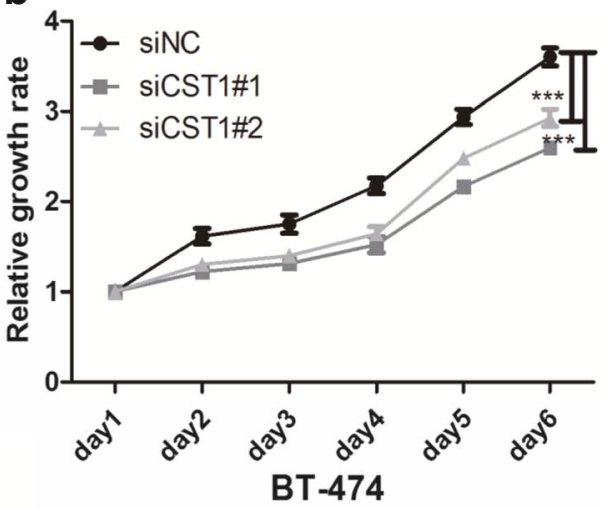

d

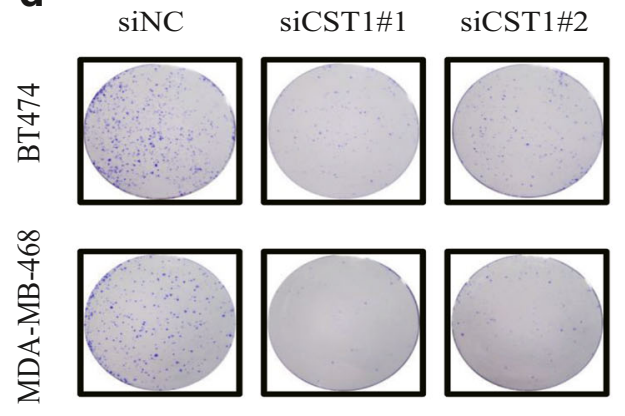

f
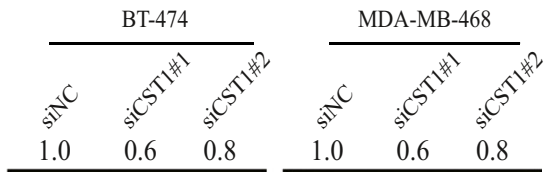

Cyclin E

$\begin{array}{lll}1.0 & 0.6 & 0.8\end{array}$
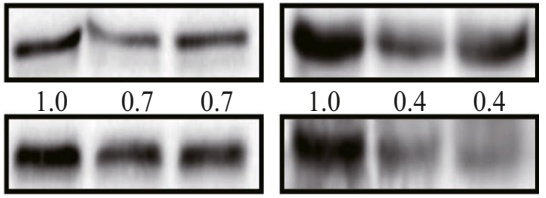

$\beta$-actin
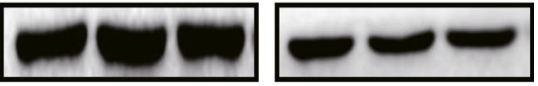

Publisher's note Springer Nature remains neutral with regard to jurisdictional claims in published maps and institutional affiliations. 